\title{
ABORDAGENS PROJETUAIS DO DESIGN E OS PROCESSOS PROJETUAIS NA ARQUITETURA
}

\author{
Mariana Soldan Hugo \\ Centro Universitário Ritter dos Reis \\ mariana.hugo@gmail.com \\ Vinicius Gadis Ribeiro \\ Centro Universitário Ritter dos Reis \\ vinicius@uniritter.edu.br
}

Resumo: Muito vem se discutindo a respeito das abordagens e metodologias projetuais na área do design, cada vez mais, estas se direcionam no sentido de uma maior participação e involviemento do usuário ao longo do desenvolvimento do projeto. No campo da arquitetura a mesma importância deve ser dada a troca com o usuário, o que se dá principalmente através do uso de prototipagem. O presente trabalho resulta de uma pesquisa qualitativa através de pesquisa bibliográfica e entrevistas semiestruturadas buscando compreender como se dá o processo projetual na arquitetura, como os arquitetos ouvem a opinião de terceiros em relação à seus projetos. Foram identificadas diversas formas de abordagem de processo de projeto e diferentes visões a respeito das avaliações de projeto, e de como inseri-las no processo de projeto. Ficou evidente a capacidade de facilitação do processo de projeto com o uso de ferramentas de prototipação ao longo de seu desenvolvimento, indo de encontro com a abordagem do design, que tem, cada vez mais, se direcionado ao encontro da interação com o usuário.

Palavras-chave: Arquitetura, Design, Processo Projetual, Prototipagem, Feedback.

\begin{abstract}
:
Much has been discussed about the approaches and project methods in design, increasingly, these are directed towards greater participation and user involvement throughout the project development. In the field of architecture the same importance should be given to exchange with the user, which is mainly through prototyping use. This paper results from a qualitative research through literature and semi-structured interviews aiming to understand how is the project process in architecture, how architects hear the user opinion regarding their projects. Were identified several design process approaches and different views about the project appraisals and how to insert them into the design process. It was evident the facilitation capacity of the design process with the use of prototyping
\end{abstract}


tools throughout their development, going towards the design approach, which has increasingly been directed to meet the user interaction.

Keywords: Architecture, Design, Project methodology, Prototyping, Feedback.

\section{INTRODUÇÃO}

Os projetos de design, assim como os de engenharia, computação ou arquitetura, estão sendo constantemente executados, e seus resultados utilizados por clientes e ou usuários. Sem dúvida, as pessoas mais indicadas para avaliar os projetos devem ser quem utiliza os mesmos. Para essas pessoas, os projetos desenvolvidos podem ter uma leitura e significados completamente diferentes do que imaginado pelos desenvolvedores - sejam designer, engenheiros, informatas ou arquitetos. Além disso, o uso e as necessidades dos usuários também podem ser mais bem avaliados por eles mesmos do que por terceiros. Dessa forma, fica evidente a importância de ouvir a opinião destas pessoas a respeito de tudo que esta sendo e foi desenvolvido para o uso das mesmas. Nos casos em que os projetos já foram executados e estão em uso, fica fácil a avaliação dos mesmos: basta que seja dada a devida importância para as suas opiniões e feedbacks.

No entanto, as opiniões e feedbacks de usuários também podem ser incorporados ao longo do processo de projeto. Dessa forma, pode-se buscar aumentar eficácia e a qualidade do resultado final do projeto. Este tema vem sendo amplamente discutido no design, autores como Cross (2008); Brown (2008) e Paton, Dorst (2011); discutem a respeito de processos projetuais e da importância de uma correta compreensão do problema projetual. Ao mesmo tempo em que autores, e instituições como Cross (2011), The

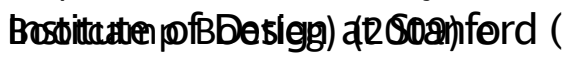
IDEO (2013), abordam metodologias projetuais e ferramentas de projeto para que os usuários possam ser envolvidos nos processos, afim de alcançar os objetivos citados acima.

Na Arquitetura as incrementações no processo projetual em busca de uma maior inserção, ou participação do usuário, já não são tão discutidas. A partir da discussão desenvolvida pelos designers, trazendo uma nova abordagem projetual, afim de se aproximar do usuário, o objetivo do trabalho foi averiguar a metodologia projetual adotada por arquitetos, de que forma os arquitetos avaliam seus projetos. 0 trabalho aborda a importância de testar e experimentar rapidamente uma ideia, antes mesmo de desenvolve-la. Busca averiguar se existe prototipagem no processo de projeto de arquitetura, em que fases de projeto se encontra e se funciona como um teste para prever futuras falhas de projeto.

Para tanto, foi conduzida uma pesquisa qualitativa, através de pesquisa bibliográfica, e uma pesquisa de campo, envolvendo dois participantes. Os participantes responderam a uma entrevista semi-estruturada, que buscou compreender de forma profunda como se dá o processo projetual desenvolvido pelos profissionais. Os mesmos receberam e estavam de acordo com um Termo de Consentimento Livre e Esclarecido. Os profissionais envolvidos na pesquisa eram profissionais autônomos, considerados jovens (Arquiteto 1, 34 anos e Arquiteto 2, 38 anos). Estes foram escolhidos por trabalharem com metodologias desenvolvidas por 
eles mesmos, podendo ser adaptados ou alterados a qualquer momento, não estando "presos" a metodologias ou procedimentos pré-estabelecidos por empresas ou escritórios de maior porte

O presente trabalho está estruturado da seguinte forma: a seção dois relaciona as falhas e considerações referentes aos projetos de design. A seção três caracteriza o recorte desejado no presente trabalho: o projeto arquitetônico. A seção quatro apresenta a ótica e percepção dos respondentes no que tange ao fenômeno em estudo. Considerações finais são apresentadas na seção seguinte.

\section{AS FALHAS E A EVOLUÇÃO DOS PROJETOS DE DESIGN}

Henry Petroski (2006) trata da importância das falhas para o desenvolvimento de ideias e produtos. Sempre que há interação entre um usuário e um objeto, há espaço para aperfeiçoamento de melhoria em questões que podem ser observadas. "Por mais que um sistema seja bem desenvolvido, nunca terá limitações de aperfeiçoamento" (PETROSKI 2006, p.03). Dessa forma, o autor aborda um conceito de extrema relevância para o design.

Percebe-se a necessidade da observação e inserção dos testes e avaliações com foco nos pontos em que poderiam ser melhor desenvolvidos durante o processo de design. Assim como as limitações podem servir como um ponto de partida para um projeto, também devem ser alvo de atenção durante o andamento do projeto, para que possam ser eliminadas - ou, pelo menos, reduzidas. Petroski (2006) vê o emprego de protótipos em parte do desenvolvimento de projetos como uma grande oportunidade de encontrar - e, assim, solucionar antecipadamente - possíveis falhas do projeto. Para o autor, protótipos são uma ferramenta que possibilita aos possíveis usuários visualizar o projeto de forma tangível.

Diversos autores, tais como, Cross (2008); Brown (2008); Paton, Dorst (2011); e Schirinner (2013), ao definirem o processo de projeto do design, evidenciam a importância de loopings de avaliação durante o desenvolvimento da proposta. Após ter-se a fase inicial de ideação, deve-se buscar feedback de usuários, antes do desenvolvimento técnico do projeto. Segundo Cross (2008), muitos modelos descritivos do design já foram criados, e pode-se observar algumas questões que se repetem entre os mesmos.

\footnotetext{
Normalmente, os modelos descritivos de design identificam a importância de gerar uma solução conceitual no início do processo, refletindo assim a natureza do foco na solução do pensamento do designer. Esta conjectura de soluções iniciais é submetida a análise, avaliação, refinamento e desenvolvimento. Às vezes, é claro, a análise e avaliação mostram falhas fundamentais na conjectura inicial, que precisa ser abandonada, um novo conceito precisa ser gerado e o ciclo reinicia. $O$ processo é heurístico: usando experiências anteriores e guias gerais que encaminham para o que parece ser o caminho certo, mas sem garantia de sucesso. (CROSS 2008 p.29)
}

Para Cross (2008) os modelos de design são frequentemente desenhados através de diagramas, mostrando suas quatro fases principais (exploração, geração, avaliação e comunicação) em uma sequência de um estágio para o outro - porém, com verificações intermediárias e retornos a fases anteriores muitas vezes necessárias. 0 uso de protótipos pode facilitar e qualificar o processo de avaliação. Como já 
comentado anteriormente, na visão de Petroski (2006), os protótipos possibilitam uma visão tangível dos projetos, possibilitando tanto uma visão concreta para avaliação dos usuários quanto para visualização pelos próprios designers.

$\mathrm{Na}$ arquitetura, esse fenômeno é muito similar. Kowaltowski et al. (2011) abordam a complexidade de um projeto arquitetônico, que envolve soluções técnicas e artísticas. Para os autores, o projeto de arquitetura é "resultado da manipulação criativa de diferentes elementos, como funções, volume, espaço, textura, luz, materiais, componentes técnicos e custos, desempenho e tecnologia construtiva" (KOWALTOWSKI ET AL. 2011 p. 21). Segundo os autores, devido a complexidade envolvida no desenvolvimento de um projeto arquitetônico, a equipe de projetos tornou-se multidisciplinar, com a participação de profissionais de diferentes áreas.

\section{Devido a complexidade do processo de concepção em arquitetura, não existem fórmulas precisas ou fixas que unam forma, função e tecnologia. O profissional aplica o conhecimento, a experiência e a compreensão do problema de maneira heurística, na busca da melhor solução, que nem sempre é alcançada. (KOWALTOWSKI ET AL. 2011 p. 54).}

Diante da complexidade de um projeto de arquitetura e de sua natureza multidisciplinar, a prototipagem pode ser uma ferramenta facilitadora valiosa durante o desenvolvimento do projeto. A comunicação entre os diferentes tipos de profissionais envolvidos, especializados em diversas áreas, pode ser esclarecida e evidenciada pelo uso dos protótipos. Cada um dos profissionais possui um diferente ponto de vista em relação ao projeto: muitas vezes, a inserção dos diferentes aspectos em um mesmo objeto gera pontos de conflito que são dificilmente identificados e compreendidos. Nesse aspecto, pode-se reforçar o uso dos protótipos como ferramenta de desenvolvimento de projeto, e pode se tornar fundamental para a busca de soluções estratégicas antecipadas - e não soluções tardias, que poderiam vir a prejudicar o resultado do projeto como um todo.

\section{O PROCESSO DE PROJETO ARQUITETÔNICO}

Segundo De Andrade, Ruschel e Moreira (2011) não existe uma versão de métodos de projeto que seja unanimidade entre os projetistas. Apesar disso, segundo os autores, pode-se dividir o processo de projeto arquitetônico em três fases, análise, síntese, avaliação e representação. $\mathrm{Na}$ análise, são definidos os principais objetivos e metas de projeto, os principais critérios de desempenho do edifício e as principais restrições. É a fase de identificação dos elementos que constituem o problema. A síntese esta associada a fase criativa do processo, onde são concebidas as ideias e soluções para os objetivos definidos na fase de análise. A fase de avaliação visa garantir que uma solução proposta seja a mais aceitável: para isso, todos os aspectos do projeto são comparados com os pré-requisitos definidos na fase inicial. Normalmente, é necessário optar-se por privilegiar algum dos itens descritos no briefing em relação a outros: nesse caso, esse equilíbrio deve ser verificado com cautela na fase de avaliação. A última fase é a de representação, esta etapa é fundamental para a execução do projeto de acordo com o que foi imaginado pelos projetistas: é a fase de comunicação do projeto. 
Para os autores, a metodologia projetual pode ser alterada de acordo com cada profissional e com cada situação. Apesar disso, algumas atividades e habilidades são comumente encontradas em boas práticas profissionais. Uma delas esta vinculada à formulação do problema e à escolha do ponto de vista. Observa-se ambas questões estão relacionadas diretamente, já que deverá ser escolhido um determinado ponto de vista pelo qual o projetista abordará o problema e, dessa forma, o redefinirá. Outra habilidade comum entre os bons arquitetos atuantes é a capacidade de comunicação, através de representações de ideias. "A habilidade de representação está na capacidade de manipulação das técnicas e na seleção das formas de representação que melhor contribuam para a compreensão do problema e para o desenvolvimento da solução do projeto" (DE ANDRADE, RUSCHEL E MOREIRA, 2011 p.98).

Além da visão dos autores citados acima sobre o processo de projeto arquitetônico, esse trabalho traz as propostas de processo idealizadas por duas entidades líderes atuantes no mercado de trabalho atual, sendo uma delas uma referencia internacional, e a outra, uma referência nacional.

O Royal Institute of British Architects (RIBA) vem desenvolvendo, desde 1963, um modelo de plano de trabalho para arquitetos do Reino Unido - o qual tem sido utilizado internacionalmente. Diversas versões foram lançadas, vista a demanda pela atualização constante do plano de trabalho. O Plano de Trabalho RIBA 2013 - vigente é formado por oito estágios, numerados de zero a sete, na seguinte estrutura: (0) Definição Estratégica, (1) Preparação e Briefing, (2) Design Conceitual, (3) Desenvolvimento do Design, (4) Design Técnico, (5) Construção, (6) Entrega e Fechamento e (7) Em Uso. Ao final de cada etapa citada acima, há uma etapa de avaliação, em que é verificado o cumprimento da etapa de acordo com as necessidades definidas no briefing. Segundo os autores, houve importantes incrementos nessa versão do plano de trabalho. Um dos principais incrementos foi a inserção de um estágio anterior ao início do processo tradicional. Esse estágio foi chamado de definição estratégica e está relacionado à compreensão mais profunda do problema de design e, se for o caso, fazer questionamentos e remodelações em relação ao mesmo. Outra mudança foi a inserção de uma etapa final, focada em coleta de feedback após a entrega do projeto e da obra concluída. O RIBA considerou fundamental a existência dessa fase final e ressalta a importância de os dados levantados nesta etapa servirem de ponto de partida para próximos projetos.

\footnotetext{
Os edifícios são renovados e reutilizados ou demolidos e reciclados em um ciclo contínuo. Se os resultados das construções estão melhorando, melhores processos de briefing serão necessários. Mais importante, o feedback dos projetos concluídos deve estar disponível para informar os projetos subsequentes. O Plano de Trabalho RIBA 2013 reconhece os estágios que um projeto de edificação atravessa e promove a importância da gravação e divulgação de informações sobre os projetos concluídos. (PLANO DE TRABALHO RIBA 2013, p.04)
}

Percebe-se a importância dada para uma etapa nova, incluída ao final do projeto. Embora constitua novidade, já é discutida e abordada a necessidade de se ouvir o que os usuários do projeto pensam a respeito do mesmo, após algum período de convivência. Por mais que os projetos sejam muito bem pensados e planejados, sempre podem deixar questões a desejar, e é apenas a partir de feedbacks dos usuários que se pode identificá-las com facilidade e clareza. Também é interessante 
observar o aprofundamento proposto na etapa de briefing, que pode ser caracterizado como mais uma tentativa de aproximar os arquitetos dos usuários e de questões específicas de projeto. Essa busca pela aproximação - criando espaço para ouvir o usuário - deve trazer aperfeiçoamentos em relação a qualidade dos projetos do ponto de vista das necessidades e valores de quem usufrui dos mesmos.

No entanto, nada fica explicitado em relação a participação do usuário ao longo do desenvolvimento do projeto. De acordo com o plano de trabalho desenvolvido pelo RIBA, esses seriam os momentos de avaliação na finalização de cada etapa de projeto. Porém, tais etapas podem ter caráter técnico - tornando a compreensão do projeto por parte do usuário uma questão bastante subjetiva, não permitindo que a visibilidade do projeto se torne tangível. Assim, o cliente e o usuário, muitas vezes, só conseguem ver - omitir suas opiniões e impressões - depois de o projeto estar finalizado e a obra concluída.

O Instituto dos Arquitetos do Brasil (IAB) disponibilizou um Roteiro para desenvolvimento do projeto de arquitetura da edificação. $O$ roteiro também divide $O$ processo de projeto a ser desenvolvido pelos arquitetos em etapas. Para o IAB, 0 projeto de arquitetura é desenvolvido em quatro fases, sendo elas: Estudo Preliminar, Anteprojeto e / ou Projeto de Aprovação, Projeto de Execução e Assistência à Execução da Obra. Cada fase tem como resultado um bloco de informações a ser avaliado e confrontado com o programa de necessidades. Apenas se pode seguir para uma próxima fase, após as reformulações necessárias à concretização dos objetivos estabelecidos no programa de necessidades. Assim, após finalizada uma etapa, as decisões tomadas não deverão ser alteradas - apenas aprofundadas e evoluídas nas etapas seguintes.

\footnotetext{
O objetivo principal do Projeto de Arquitetura da Edificação é a Execução da Obra idealizada pelo arquiteto. Essa obra deve se adequar aos contextos naturais e culturais em que se insere e responder às necessidades do cliente e futuros usuários do edifício. As exigências do cliente e usuários se exprimem através do programa de necessidades que define metodicamente o objetivo do projeto. (IAB, 2013 p.01)
}

No processo sugerido pelo IAB (2014) não há etapa de definição do problema. No ponto de vista do IAB, o problema deve ser identificado e discriminado em uma atividade anterior ao projeto, não sendo de responsabilidade do arquiteto (IAB, 2014). $O$ trabalho do arquiteto seria desenvolver um projeto que suprisse as necessidades definidas e entregues a ele já de forma pré-definida. Uma questão importante das teorias de processo de projeto desenvolvidas é a importância e reconhecimento que é dado para as avaliações e looping durante as fases iniciais de projeto - inclusive, na fase de definição do problema.

É interessante perceber como duas questões que surgem como diferença entre os dois modelos propostos para projetos de arquitetura - como, por exemplo, itens que estão excluídos no modelo do IAB mas não estão incluídos no modelo do RIBA -, foram justamente os incrementos realizados na última atualização feita pelo RIBA. Tais incrementos estão ligados à fase de identificação do problema e à fase de ouvir - ou feedback. Sendo assim, é possível que seja uma tendência de caminho de evolução nos processos de arquitetura: nesse sentido, se aproximando-se das teorias e conceitos desenvolvidos já anteriormente pelo design. 
Segundo Petroski (2006), "O passado é um repositório de diversos tipos de falhas já cometidas, se levadas em conta, podem servir de lição para futuros projetos" (PETROSKI 2006, p.169). Esta afirmação reitera a importância das falhas cometidas anteriormente, a necessidade de incluir feedbacks nos processos de projeto fica cada vez mais evidente. Assim, ouvir a opinião dos usuários antes e durante a condução do projeto pode ser a melhor maneira de se identificar falhas a serem evitadas em futuros projetos.

\section{O PROCESSO DE PROJETO ARQUITETÔNICO SEGUNDO PROFISSIONAIS}

Entendeu-se necessário acrescentar ao estudo a metodologia de trabalho utilizada por profissionais atuantes na área de arquitetura. Dois arquitetos foram entrevistados a respeito de seus processos de desenvolvimento de projeto denomindos aqui como Arquiteto 1 e Arquiteto 2.

O processo de trabalho descrito pelo Arquiteto 1 é similar aos processos de arquitetura idealizados pelas entidades e descritos na seção dois. Segundo o profissional, o modelo de desenvolvimento de projeto é dividido em cinco etapas. Sendo elas, briefing, estudo preliminar, anteprojeto, projeto executivo e assistência a execução. O profissional explica que só se inicia uma próxima etapa perante a aprovação do cliente a respeito da etapa anterior.

A primeira etapa é a de brief: o Arquiteto 1 busca conversar com o cliente com muitas interações - para compreender profundamente seus desejos, necessidades e prioridades. Segundo o respondente, esse processo de conhecimento se dá, em média, ao longo de três encontros. Na fase de brief, além de conhecer o cliente e suas necessidades, o profissional também conhece o lugar onde o projeto será desenvolvido.

$\mathrm{Na}$ segunda etapa, chamada de estudo preliminar, o arquiteto lança e desenvolve o projeto até um nível bem avançado, onde o mesmo já pode ser visualizado como algo concreto praticamente em sua forma final. O Arquiteto 1 comenta que usa uma estratégia de não dividir com o cliente suas ideias iniciais antes de tê-las desenvolvido e concretizado de alguma forma. O profissional comenta que prefere não mostrar esquemas conceituais e plantas abstratas, pois estas ferramentas são de difícil compreensão por parte do cliente, que pode ficar inseguro e acabar fazendo uma avaliação confusa a respeito do projeto. Para aumentar a chance de encantamento por parte do cliente, o arquiteto desenvolve o projeto até chegar em um protótipo físico ou eletrônico de alta qualidade, que é apresentado na reunião de estudo preliminar. Com o uso dessas ferramentas, o cliente compreende plenamente o conceito proposto e consegue dar um feedback bastante realista e eficiente a respeito do projeto.

A terceira etapa é o anteprojeto, onde é desenvolvido e revisado o estudo preliminar a partir do feedback recebido na reunião com o cliente. Para as apresentações seguintes, o material produzido já não é mais tão elaborado, pois o cliente já possui a ideia geral do projeto e sua impressão formada, sendo necessários somente modificações de trechos específicos. A quarta etapa é o projeto executivo: nessa etapa, o projeto já está definido e aprovado pelo cliente de forma geral e é desenvolvida apenas a parte técnica e de representação para a execução. São contatados fornecedores e definidos detalhes técnicos e construtivos. Segundo o Arquiteto 1 , após o projeto executivo é que se assiste a execução. $O$ arquiteto 
comenta que prefere se envolver com esta etapa, para garantir a aplicação das soluções definidas em projeto.

O Arquiteto 2 relata uma metodologia de projeto baseada em três etapas de trabalho. Para ele o projeto se divide em estudo preliminar, anteprojeto e projeto executivo. A primeira, de estudo preliminar, se inicial com o briefing. 0 Arquiteto 2 comenta que este se dá através de uma conversa inicial com o cliente e, a partir disso, desenvolve uma etapa de trabalho interna no escritório de verificação de compatibilidade das informações passadas pelo cliente, verificando se o programa de necessidades está de acordo com a área que o cliente solicitou - e, da mesma forma, se a área desejada e padrão de construção estão de acordo com o investimento pretendido pelo cliente. Tendo acordado com os arquitetos, iniciam a geração de ideias - porém, havendo alguma incoerência, pelo menos uma nova reunião de alinhamento ainda se faz necessária.

A etapa de estudo preliminar, de acordo com o Arquiteto 2, segue com o lançamento de ideias. $\mathrm{O}$ arquiteto e sua equipe buscam estratégias utilizadas por eles em projetos anteriores similares; muitas vezes, tais estratégias são aplicadas novamente, ou a partir de uma delas é gerada uma nova estratégia específica para o projeto. Os profissionais começam a desenhar o terreno com suas dimensões e topografia específicas para atuar como uma base. Sobre essa base, começam a lançar um zoneamento com as áreas, de acordo com o programa de necessidades e sua conexões e posicionamentos. Juntamente com isso esse zoneamento, começam a surgir estudos de volumetria, estudos de composição e formatos. Durante o desenvolvimento dos estudos de forma e composição, são levadas em conta a estratégia escolhida e as referencias de linguagem arquitetônica utilizadas pelo escritório. O Arquiteto 2 comenta que, nesse desenvolvimento, já são adotadas estratégias de solução para instalações e estrutura. Quando se chega a uma primeira ideia, é montada uma modelagem eletrônica rápida. Ao chegar a uma ideia volumétrica satisfatória no ponto de vista dos arquitetos, já se pode apresentar um mockup ao cliente, a fim de obter o seu retorno. O Arquiteto 2 comenta que eles optam por não mostrar desenhos em duas dimensões para o cliente.

Após a reunião de apresentação do estudo preliminar - onde os arquitetos escutam o feedback do cliente referente a etapa específica - são feitas as alterações no projeto sobre do modelo $3 \mathrm{D}$, ao mesmo tempo em que mais características de materialidade e definições são incorporadas no modelo. Dessa forma, o projeto vai gradativamente sendo desenvolvido e materializado, sofrendo aos poucos a interferência do cliente e se adaptando de acordo com suas impressões. Após um bom desenvolvimento do projeto a partir deste método, chega-se ao anteprojeto já desenvolvido e aprovado pelo cliente. A partir dai, é desenvolvido o projeto executivo. Nessa etapa existe pouca participação do cliente, sendo a fase de desenhos técnicos e de troca de informação com outros técnicos.

Foi desenvolvido um quadro comparativo (Quadro 1) contendo as duas metodologias idealizadas pelos institutos, apresentados na seção 3, e as metodologias relatadas pelos profissionais entrevistados.

A Figura 1 foi desenvolvida para facilitar a visualização e compreensão do Quadro 1. A proposta é possibilitar uma visualização mais clara das diferenças quanto à abrangência de cada metodologia nas fases de pré e pós projeto. Dentre as metodologias abordadas, a apresentada pelo RIBA (2013), contém a maior variedade 
de temáticas, possuindo duas etapas anteriores ao desenvolvimento do projeto em si e três posteriores. Desta forma serviu como base para a concepção da Figura 1 que define uma cor para cada conteúdo.

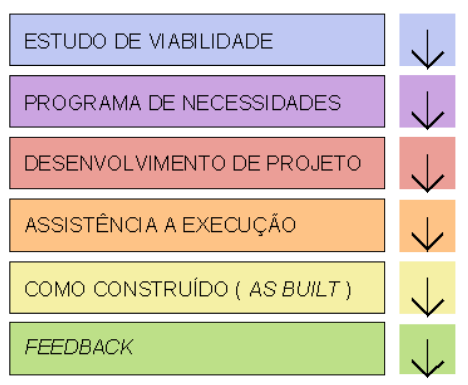

Figura 1 - Síntese de conteúdos abordados pelas metodologias analisadas.

Fonte: Desenvolvido pelos autores.

No Quadro 1, a seguir, as metodologias foram divididas em etapas, conforme descrições dos institutos e participantes. A cada etapa foi atribuida uma cor, de acordo com o conteúdo abordado na mesma, de acordo com a Figura 1. Dessa forma, é possível visualizar a abrangência de conteúdo de cada uma das metodologias, pela presença ou não de todas as cores. Quando são demonstrados etapas com a mesma cor, significa que são subdivisões da mesma dentro do processo, abordando um mesmo conteúdo.

\begin{tabular}{|c|c|c|c|}
\hline RIBA (2013) & IAB (2014) & ARQUITETO 1 & ARQUITETO 2 \\
\hline DEFINIÇÁO ESTRATEGICA & & & \\
\hline BRIEF & & BRIEF & \\
\hline DESIGN CONCEITUAL & ESTUDO PRELIMINAR & ESTUDO PRELIMINAR & ESTUDO PRELIMINAR \\
DESENVOLVIMENTO & ANTEPROJETO & ANTEPROJETO & ANTEPROJETO \\
DESIGN TÉCNICO & PROJETO DA EXECUÇÄO & PROJETO EXECUTIVO & PROJETO EXECUTIVO \\
\hline ASSISTENCIA EXECUCAAO & ASSISTENCIA EXECUÇÄO & ASSISTENCIA EXECUÇAO & \\
\hline ENTREGAE FECHAMENTO & & & \\
\hline EM USO & & & \\
\hline
\end{tabular}

Quadro 1 - Síntese de conteúdos abordados pelas metodologias analisadas.

Fonte: Desenvolvido pelos autores.

De modo geral, ao observar-se o Quadro 1, percebe-se que a fase de desenvolvimento de projeto, representada em em vermelho, é bastante similar e central em todas as metodologias analisadas. No entanto, algumas metodologias apresentam mais etapas antes e depois desta fase central. Conforme comentado anteriormente, a metodologia idealizada pelo RIBA (2013) é a mais abrangente, incluindo como parte do projeto, duas etapas anteriores e três etapas posteriores ao desenvolvimento de projeto em si. Para o Arquiteto 1, a etapa de brief e a assistencia a execução, são incluidas no processo como etapas distintas do desenvolvimento. Para o IAB (2014) além do desenvolvimento em si, existe a assistência a execução. E o processo relatado pelo Arquiteto 2, consiste somente da fase central, de desenvolvimento. 
Assim, algumas metodologias propõem etapas de preparação e investigação do problema separadamente, antes do desenvolvimento do projeto em si, assim como etapas específicas de feedbacks após o desenvolvimento do projeto, para que possíveis problemas possam ser estudados e evitados em futuros projetos.

No entanto, de acordo com a descrição das etapas, pode-se perceber que algumas atividades que seriam desenvolvidas nestas etapas anteriores e posteriores ao desenvolvimento, as vezes, estão presentes ao longo do mesmo. A etapa de brief, no caso do Arquiteto 2, por exemplo, foi incorporada à etapa de estudo preliminar. Desta forma, mesmo a atividade não tendo sido citada como uma etapa distinta, ela esta presente na metodologia adotada.

Segundo o Arquiteto 2, o processo de projeto se encontra em uma fase de transição, pois seu escritório esta iniciando atualização de uma nova ferramenta. Esta ferramenta é chamada de modelagem da informação da construção (Building Modeling Information, da BIM). Segundo o profissional, esta ferramenta transforma o processo em algo muito similar a uma prototipagem constante. Desde as fases bastante iniciais do projeto, as informações vão sendo incorporadas ao programa e o projeto vai sendo visualizado em 3D. Segundo o arquiteto, essa ferramenta proporciona prototipagem muito rápida, e assim muitas ideias são facilmente testadas e avaliadas em 3D rapidamente, tanto pelos próprios arquitetos quanto pelo cliente. Para o arquiteto, a ferramenta está apoiando a visualização de futuros problemas de projeto e, com isso, soluções podem ser refletidas em fases iniciais do desenvolvimento, gerando menores interferências em outras questões ou em fases futuras.

O arquiteto comenta que essa ferramenta de prototipagem também está facilitando a comunicação com outros profissionais que estejam trabalhando no mesmo projeto - porém em questões de caráter mais técnico específico, como estrutura, projeto hidrossanitário etc. O BMI possibilita a visualização fácil de possíveis interferências dos diferentes projetos.

Segundo De Andrade e Ruschel (2011) o processo de projeto arquitetônico deve ser reestruturado a fim de incluir o BIM. Para os autores, pode-se ganhar muito com a utilização desta ferramenta: porém, se a mesma facilita por um lado, traz complexidade em novos fatores ainda não experimentados. Por isso, recomendam que os profissionais que optarem pela utilização da ferramenta precisam estar preparados para adaptarem suas metodologias, assim como avaliar e testar novas metodologias até encontrar uma que julguem apropriada para a nova situação.

Contudo, prototipagens para feedbacks e trocas com usuários são fundamentais para o desenvolvimento de projetos de sucesso, estes podem ser adotados em diferentes formas e etapas de projeto. Antes, durante e depois da execução do mesmo.

\section{CONSIDERAÇÕES FINAIS}

Conforme as discussões que vem sendo desenvolvidas no campo do design, é fundamental a observação de falhas e questões que podem ser aprimoradas nos projetos para o desenvolvimento dos mesmos, assim como, para o desenvolvimento de novos projetos. A prototipagem pode ser utilizada para a obtenção de feedbacks tanto por parte dos usuários quanto dos próprios profissionais envolvidos no projeto. 
Os feedbacks são uma excelente forma de identificar falhas nos projetos. Isso pode ocorrer tanto durante o projeto quanto após a sua finalização e execução.

Após observar-se as diversas versões a respeito do projeto arquitetônico, conclui-se que não existe uma versão definida como verdade única e ideal em relação ao processo de projeto arquitetônico. Cada profissional adota uma abordagem que pode variar de acordo com as experiências vividas pelo mesmo - assim como, de acordo com as necessidades e característica específicas de cada projeto. Apesar disso, diversas características e atividades do processo se repetem de acordo com as fontes consultadas, assim como com as entrevistas realizadas com profissionais da área.

Pode-se identificar diferentes formas adotadas pelos arquitetos para a avaliação de seus projetos, tanto durante o desenvolvimento, quanto após a finalização da execução do mesmo. Para o RIBA (2014), não fica explícito o uso de prototipagem durante o processo de projeto para a facilitação de avaliação do mesmo; no entanto, é dado um grande enfoque na importância da avaliação após ocupação e utilização do edifício. Esse enfoque busca evitar a repetição de erros anteriores em futuros projetos (RIBA, 2014). Na descrição do processo pelo IAB (2014), não é sugerida não é sugerida uma etapa de avaliação do projeto após a execução do mesmo.

No entanto, ao observarmos os relatos dos profissionais entrevistados, fica clara a importância dada por eles ao uso de protótipos - ainda que virtuais - durante o processo de desenvolvimento de projeto. Mesmo que de formas diferenciadas, e com estratégias diferenciadas, os dois profissionais utilizam o protótipo como forma de uma comunicação eficiente com o cliente - em busca de um feedback sólido e seguro do mesmo - já nas fases iniciais de projeto, o que não seria possível sem o uso desta ferramenta.

O Arquiteto 2 aborda outro uso importante para o qual ele adota o protótipo: para a avaliação e busca de falhas no projeto, também focando em levantar as falhas nas etapas iniciais do processo. A prototipagem se mostrou uma ferramenta essencial para este profissional - tanto como uma ferramenta interna de desenvolvimento de projeto, quanto como uma ferramenta de comunicação entre os diversos projetistas envolvidos no trabalho.

No entanto, nenhum dos profissionais entrevistados relatou sobre a importância de se ouvir e buscar informações a respeito de seus projetos já ocupados e que estão sendo utilizados, a fim de buscar melhorias para os seus próximos projetos. Quanto a este aspecto é interessante observar que a inclusão desta etapa foi uma mudança recente feita no processo sugerido pelo RIBA (2014), não sendo ainda presente na metodologia indicada pelo IAB (2014).

\section{REFERÊNCIAS}

BROWN, T. Design thinking. Harvard Business Review, 2008.

CERVO, A. BERVIAN, P. SILVA, R. Metodologia Científica. São Paulo : Prentice Hall, 2007.

CRESWELL, J. Projeto de pesquisa : métodos qualitativo, quantitativo e misto. Porto Alegre : Artmed, 2010.

CROSS, N. Designerly ways of knowing. 4th ed. Londres, UK. Springer- Verlag, 2007.

CROSS, N. Design Thinking: Understanding how designers think and work, Berg, 
Oxford, 2011.

CROSS, N. Engeneering design methods: Strategies for Product Design, Chichester: John Wiley \& Sons, 2008.

CROSS, N., CHRISTIAANS, H. \& DORST, K. (eds) Analysing Design Activity. Chichester: Wiley, 1996. DORST, K. The nature of design thinking, 2010. Disponível em: $<$ http://dab.uts.edu. au/research/conferences/dtrs8/docs/DTRS8-Dorst.pdf >. Acesso em 06 jun. 2013.

DE ANDRADE, M. RUSCHEL, R. O processo de projeto em arquitetura. Capítulo 21: Building Information Modeling (BIM) São Paulo: Oficina de Textos, 2011.

IDEO. DCH: Kit de Ferramentas http://www.ideo.com/work/human-centered-Designtoolkit/ 25.10.2013.

INSTITUTE OF DESIGN AT STANFORD. Bootcamp Bootleg. Stanford, 2009 http://dschool.stanford.edu/blog/2009/12/16/the-bootcamp-bootleg-is-here/ 10.11.2014

INSTITUTO DOS ARQUITETOS DO BRASIL (IAB). Roteiro para desenvolvimento do projeto de Arquitetura da edificação (http://www.iab.org.br/documentos 15.09.2014).

KOWALTOWSKI, D.; MIKAMI, S. A.; PINA, G.; PRATA, A. R.; FACCIN DE CAMARGO, R. C. Ambiente construído e comportamento humano: necessidade de uma metodologia. Salvador: Encontro Nacional De Tecnologia No Ambiente Construído, 2000.

KOWALTOWSKI, D.; CELANI, G.; MOREIRA, D.; PINA, S.; RUSCHEL, R.; SILVA, V.; LABAKI, L.; PETRECHE, J. Reflexão sobre metodologias de projeto arquitetônico. Porto Alegre: Ambiente Construído v. 6, n. 2, p. 07-19, abr./jun. 2006.

KOWALTOWSKI, D. ; MOREIRA, D. ; PETRECHE, J. ; FABRICIO, M. O processo de projeto em Arquitetura. São Paulo: Oficina de Textos, 2011.

KRIPPENDORFF, K. The semantic turn: A new foundation for Design. 2006. New York, 2006: Taylor and Francis Group.

KRIPPENDORFF, K. The semantic turn: a new foundation for design. New York: Taylor\&Francis, CRC Press, 2006.

LAWSON, B. How designers think: The design process demystified. Nova lorque: Routledge, 2005.

NEUFERT, E. Arte de projetar em arquitetura, 18.ed. São Paulo: Gustavo Gili, 2013

PATON, B. DORST, K. Briefing and reframing: A situated practice. Design Studies Vol 32 No. 6. Novembro 2011.

PETROSKI, H. Sucesso através da falha: O paradoxo do design. Oxfordshire: Princeton University Press, 2006

RIBA: Plano de trabalho RIBA 2013.

(http://www.architecture.com/RIBA/Professionalsupport/RIBAOutlinePlanofWork2013 .aspx) 15.09.2014

SCHRINNER, M. What is servise architecture thinking?. Munster, 2013. Master thesis, Munster School of Architecture. 